Plant Metabolomics 
Xiaoquan Qi · Xiaoya Chen Yulan Wang

Editors

\section{Plant Metabolomics}

Methods and Applications

岩 Chemical Industry Press 型 Springer 
Editors

Xiaoquan Qi

Institute of Botany

Chinese Academy of Sciences

Beijing

China

Xiaoya Chen

Shanghai Institute for Biological Sciences

Chinese Academy of Sciences

Shanghai

China

\author{
Yulan Wang \\ Wuhan Institute of Physics and \\ Mathematics \\ Chinese Academy of Sciences \\ Wuhan, Hubei \\ China
}

$\begin{array}{lll}\text { ISBN 978-94-017-9290-5 } & \text { ISBN 978-94-017-9291-2 } & \text { (eBook) } \\ \text { DOI 10.1007/978-94-017-9291-2 } & & \end{array}$

Library of Congress Control Number: 2014951658

Springer Dordrecht Heidelberg New York London

Jointly published with Chemical Industry Press, Beijing

ISBN: 978-7-122-21436-2, Chemical Industry Press, Beijing

(C) Chemical Industry Press, Beijing and Springer Science+Business Media Dordrecht 2015

This work is subject to copyright. All rights are reserved by the Publishers, whether the whole or part of the material is concerned, specifically the rights of translation, reprinting, reuse of illustrations, recitation, broadcasting, reproduction on microfilms or in any other physical way, and transmission or information storage and retrieval, electronic adaptation, computer software, or by similar or dissimilar methodology now known or hereafter developed. Exempted from this legal reservation are brief excerpts in connection with reviews or scholarly analysis or material supplied specifically for the purpose of being entered and executed on a computer system, for exclusive use by the purchaser of the work. Duplication of this publication or parts thereof is permitted only under the provisions of the Copyright Law of the Publishers' locations, in its current version, and permission for use must always be obtained from Springer. Permissions for use may be obtained through RightsLink at the Copyright Clearance Center. Violations are liable to prosecution under the respective Copyright Law.

The use of general descriptive names, registered names, trademarks, service marks, etc. in this publication does not imply, even in the absence of a specific statement, that such names are exempt from the relevant protective laws and regulations and therefore free for general use.

While the advice and information in this book are believed to be true and accurate at the date of publication, neither the authors nor the editors nor the publishers can accept any legal responsibility for any errors or omissions that may be made. The publishers make no warranty, express or implied, with respect to the material contained herein.

Printed on acid-free paper

Springer is part of Springer Science+Business Media (www.springer.com) 


\section{Preface}

Life sciences progress quickly with each passing day. The improvement of genomics and related analytical techniques greatly promoted the rapid development of transcriptomics, proteomics, metabolomics, and phenomics. Thus, the means of system integration can be employed to reveal life phenomenon at multiple levels. The above research thoughts and methods gave birth to systems biology. Metabolomics is an important part of systems biology. Metabolites are closest to phenotype, thus the change in metabolites can more directly reveal the function of genes. And metabolic markers have important application values in the early diagnosis of diseases. There are a wide range of plant species in nature. Different groups of plant species synthesize different special compounds. It is estimated that there are $0.2-1$ million kinds of metabolites synthesized by plants. The structural and physicochemical properties vary widely, making plant metabolomics research more challenging. Since the year 2002 when the first International Plant Metabolomics Conference was held in Wageningen, analytical techniques and methods of plant metabolomics have been developing rapidly and applied in several areas, such as plant scientific research, biotechnology safety assessment, crop breeding, etc., and play important roles in the study of gene function and the analysis of metabolic pathway and metabolic network regulation. Plant metabolomics research in China started around 2005, and currently has a good development trend. This book written in cooperation by researchers active in plant metabolomics in China, not only introduces the latest advances in plant metabolomics and analyzes the development trend in the next few years, but also demonstrates new studies of authors in their respective scientific projects, reflecting the current study level of China very well.

This book includes three parts introducing and demonstrating plant metabolomics. The first part includes an overview of plant metabolomics and the principles, methods, issues, considerations, and developments of metabolite analytical technologies, which mainly include mass spectrometry and nuclear magnetic resonance; the second part includes metabolomics data analysis, metabolites determination, metabolomics database and metabolic network study; the third part includes detailed application examples in plant metabolomics, which mostly are the current research 
achievements in recent years. We strive to be realistic and practical in this book, and hope that this book can promote the rapid development of plant metabolomics in China. Immense thanks to the authors of each chapter for taking the time from busy research and teaching tasks. Many authors of this book received the funding of "Metabolism and Regulation of Special Crop Nutrients (2007CB108800)" and the funding of "The Formation Mechanism and Control Approaches of Harmful Substances in Animal Products (2009CB118800)" from "973" plans of Ministry of Science of China. This book received the funding of "Metabolism and Regulation of Special Crop Nutrients" project office, too. Warm thanks to Prof. Chun-Ming Liu from the Institute of Botany of Chinese Academy of Sciences for his concern and help in the publishing of this book. Readers are welcome to criticize and correct errors and shortcomings in this book.

November 2010

Xiaoquan Qi

Xiaoya Chen

Yulan Wang 


\section{Contents}

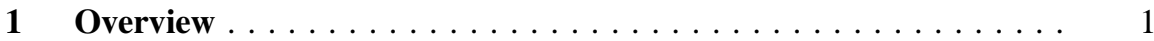

Xiaoya Chen, Xiaoquan Qi and Li-Xin Duan

2 Gas Chromatography Mass Spectrometry Coupling

Techniques......................... 25

Zhen Xue, Li-Xin Duan and Xiaoquan Qi

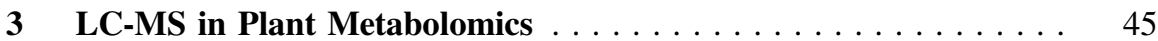

Guo-dong Wang

4 Nuclear Magnetic Resonance Techniques. . . . . . . . . . . . . . 63

Fu-Hua Hao, Wen-Xin Xu and Yulan Wang

5 Multivariate Analysis of Metabolomics Data . . . . . . . . . . 105 Jun-Fang Wu and Yulan Wang

6 Metabolomic Data Processing Based on Mass Spectrometry Platforms . . . . . . . . . . . . . . . . . . . . . . . . . 123

Tian-lu Chen and Rui Dai

7 Metabolite Qualitative Methods and the Introduction of Metabolomics Database. .

Li-Xin Duan and Xiaoquan Qi

8 Plant Metabolic Network . . . . . . . . . . . . . . . . . . . . . . . 195

Shan Lu

9 Applications of LC-MS in Plant Metabolomics. . . . . . . . . . . . 213 Guo-dong Wang 
10 Application of Metabolomics in the Identification of Chinese

Herbal Medicine. . . . . . . . . . . . . . . . . . . . . . . 227

Li-Xin Duan, Xiaoquan Qi, Min Chen and Lu-qi Huang

11 Metabolomics-Based Studies on Artemisinin Biosynthesis . . . . . . 245

Hong Wang, Hua-Hong Wang, Chen-Fei Ma, Ben-Ye Liu,

Guo-Wang $\mathrm{Xu}$ and $\mathrm{He}-\mathrm{Chun} \mathrm{Ye}$

12 NMR-Based Metabolomic Methods and Applications . . . . . . . . 275

Chao-Ni Xiao and Yulan Wang

13 Metabolomics Research of Quantitative Disease

Resistance Against Barley Leaf Rust . . . . . . . . . . . . . . . . . 303

Li-Juan Wang and Xiaoquan Qi 